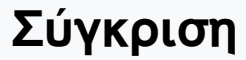

Tón. 20 (2009)

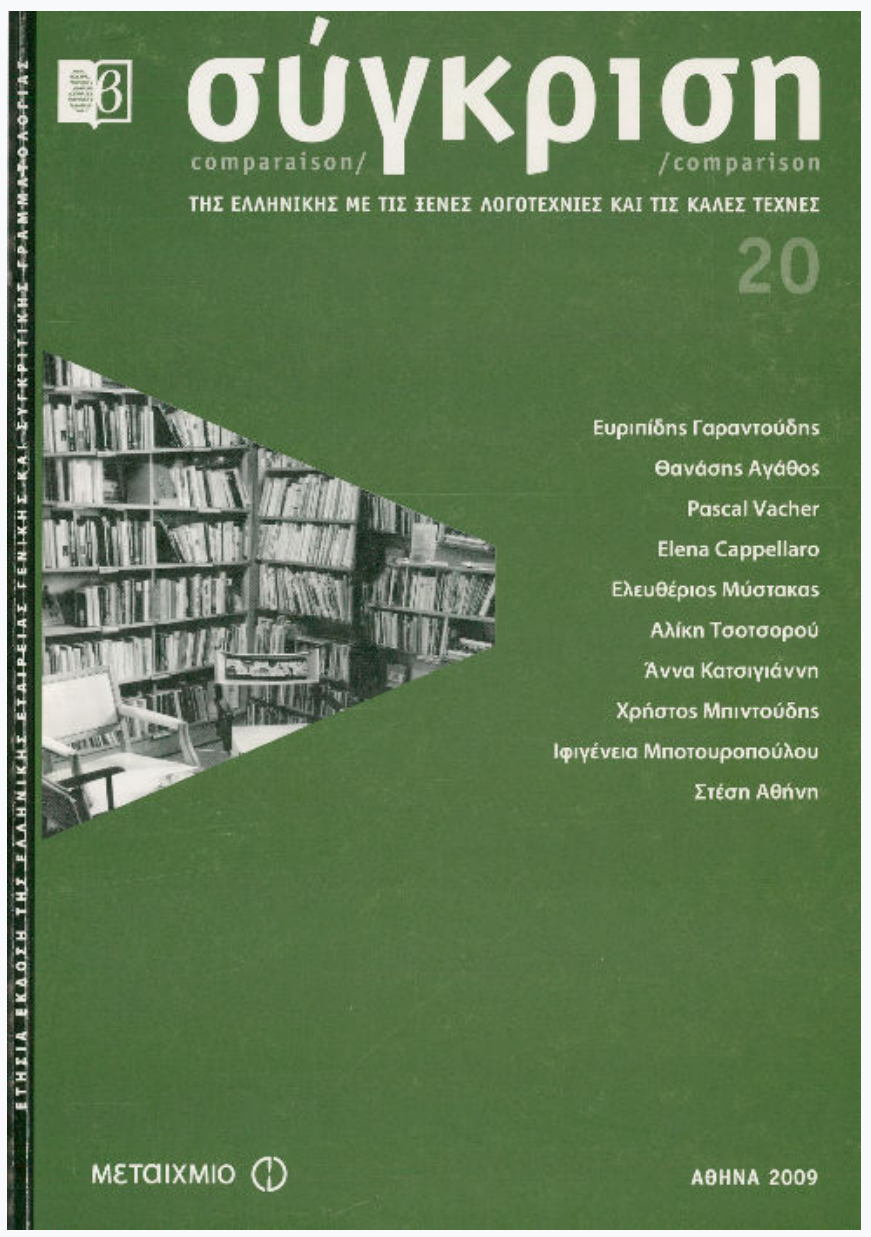

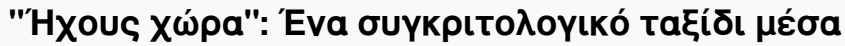

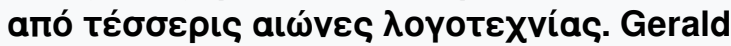
Gillespie, Echoland. Readings from Humanism to Postmodernism

\section{Eún Пвтропои́ภоu}

doi: $10.12681 /$ comparison.68

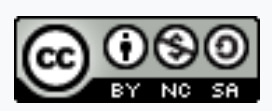

Ađ¿ıı Xpńбnৎ Creative Commons Attribution-NonCommercial-ShareAlike 4.0.

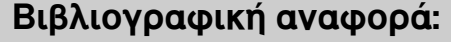

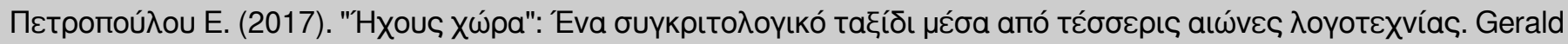

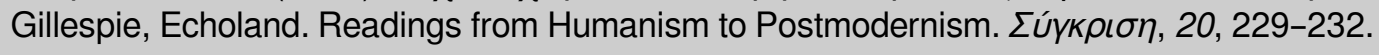

https://doi.org/10.12681/comparison.68 


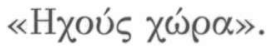

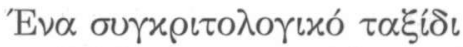

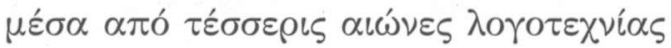

\begin{abstract}
Gerald Gillespie, Echoland. Readings from Humanism to Postmodernism, Brüssel, Bern, Berlin, Frankfurt a.M., New York, Oxford, Wien: Peter Lang,

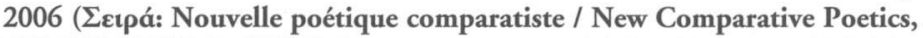

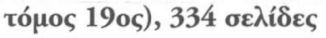

$\mathbf{T}$

ı троки́тteı оиүүрарıка́, ótav

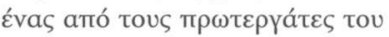

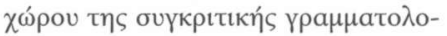

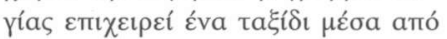

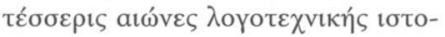

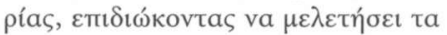

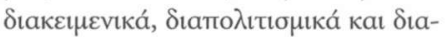

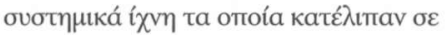

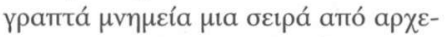

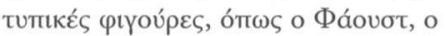

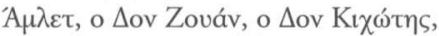

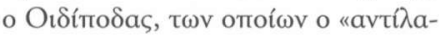

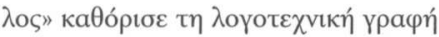

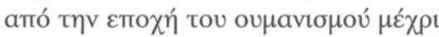

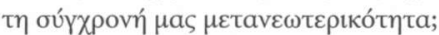

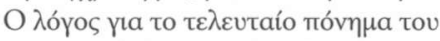

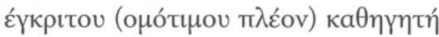

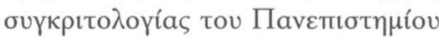

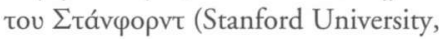
USA) Gerald Gillespie, о отоíos $\delta \varepsilon$ -

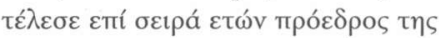

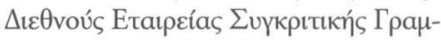

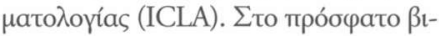

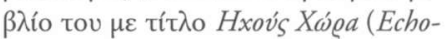
land. Readings from Humanism to Post-

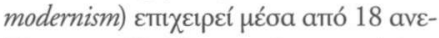

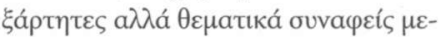

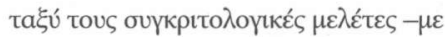

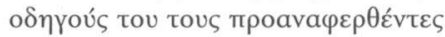

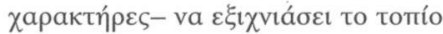

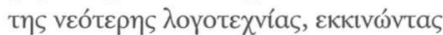

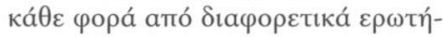

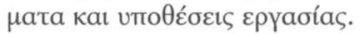

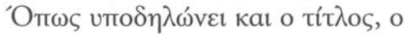

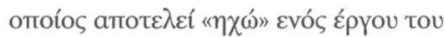

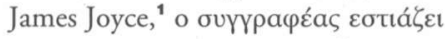

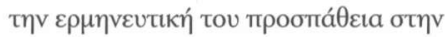

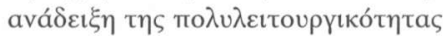

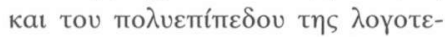

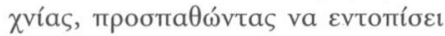

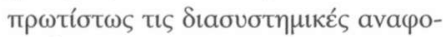

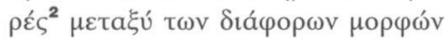

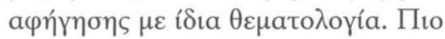

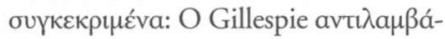

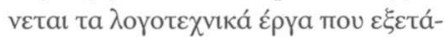

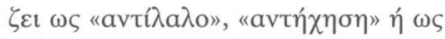

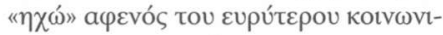

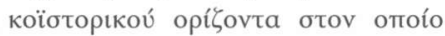

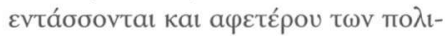

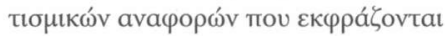

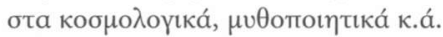

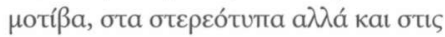

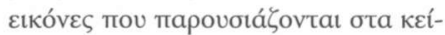

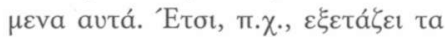




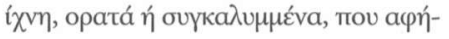

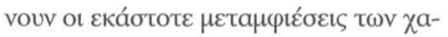
ракти́ $\omega \mathrm{v} \mathrm{\sigma т \eta} \mathrm{\lambda оүот \varepsilon \chi vเк \eta ́} \mathrm{точ \varsigma} \mathrm{\delta เa-}$

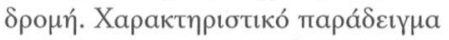

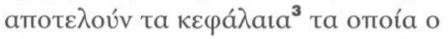
Gillespie a

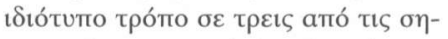

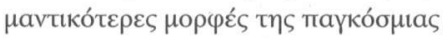

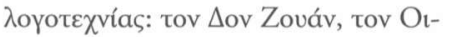

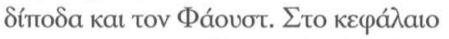

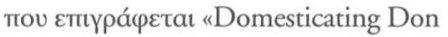
Juan», ${ }^{4}$ о Gillespie катаүра́

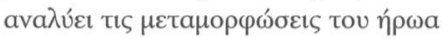

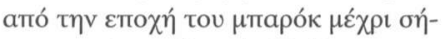
$\mu \varepsilon \rho a$, aтó tov Tirso de Molina kaı тov

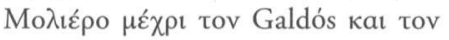

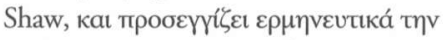

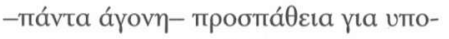

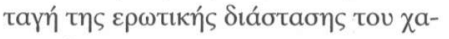
ракти́ра.

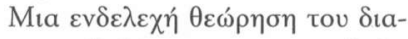

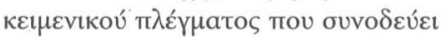

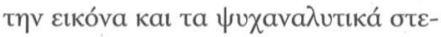

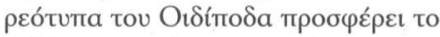

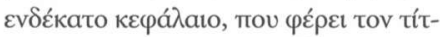
$\lambda$ "Romantic Oedipus», ${ }^{5}$ ото отоі́o

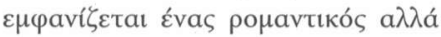

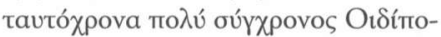

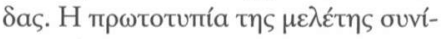

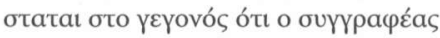

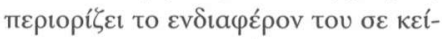

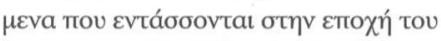

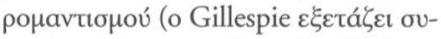

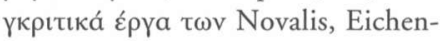
dorff, Brentano, Tieck кaı по $\lambda \lambda \omega$ v á $\lambda$ -

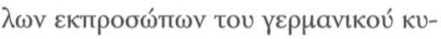

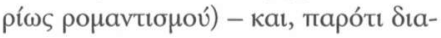

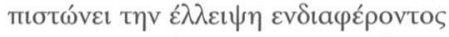

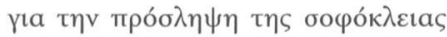

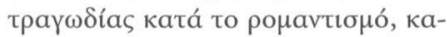

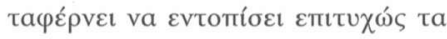

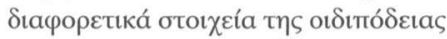

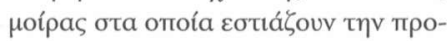

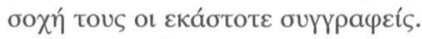

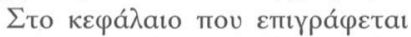

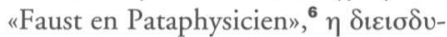

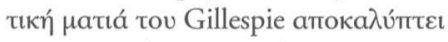

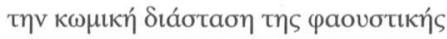

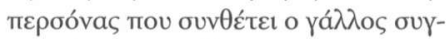

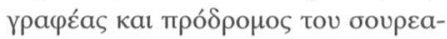

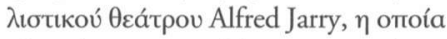

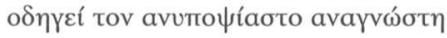

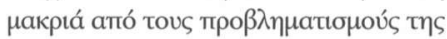

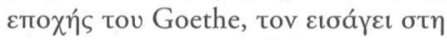

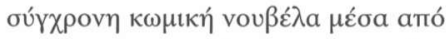

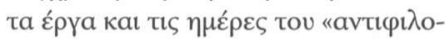
бó(pou» $\Delta \rho a$ Faustroll ${ }^{7}$ kat tov $\mu$ veí

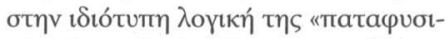

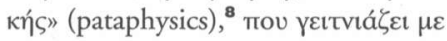

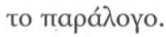

O Gillespie a

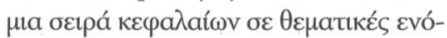

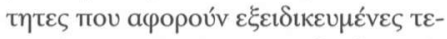

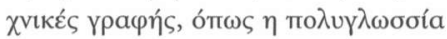

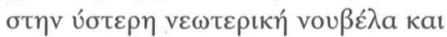

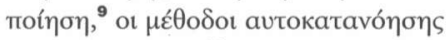

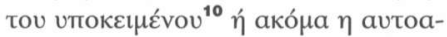

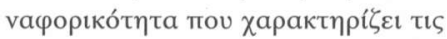

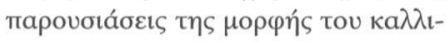

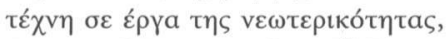

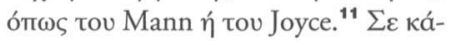

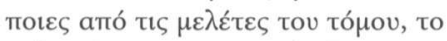

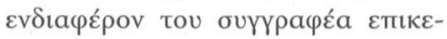

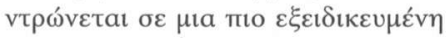




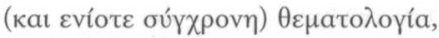

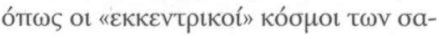

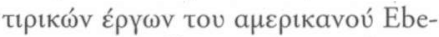

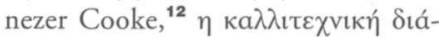

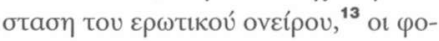

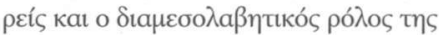

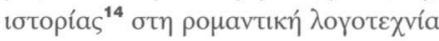

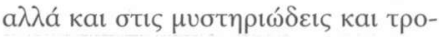

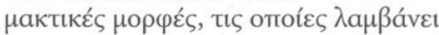

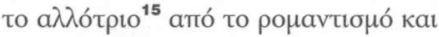

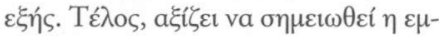

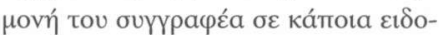

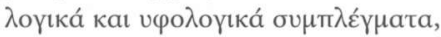

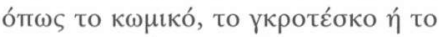

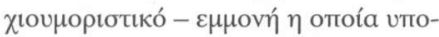


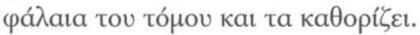

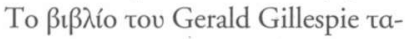

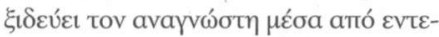

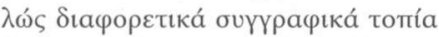

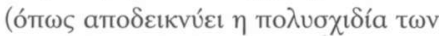

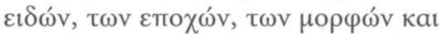

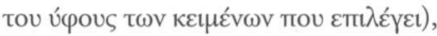

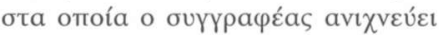

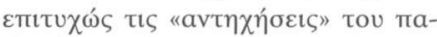

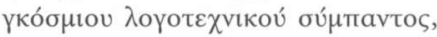

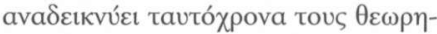

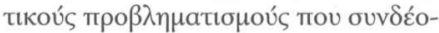

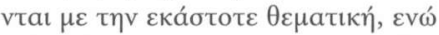

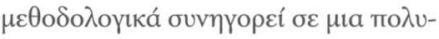

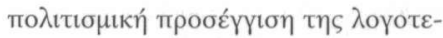

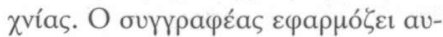

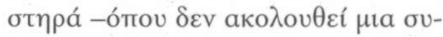

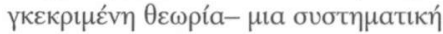

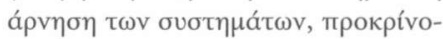

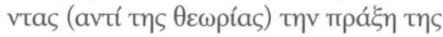

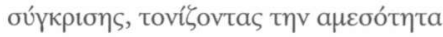

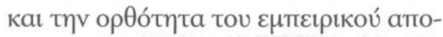

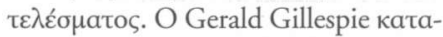

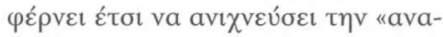

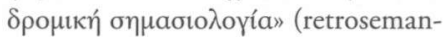

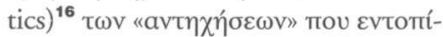

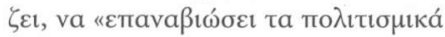

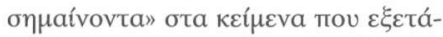

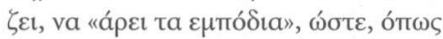

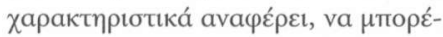

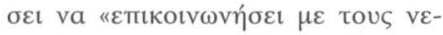

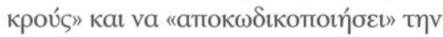

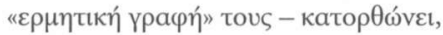

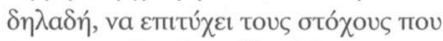

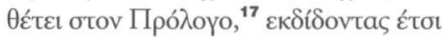

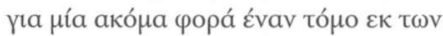

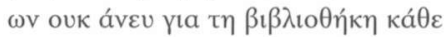
$\mu \varepsilon \lambda \varepsilon \tau \eta \tau \eta \dot{~ т \eta ~}$

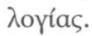

Еún Пвтротоои́ภov Пауєтเотท́

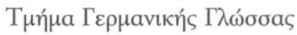

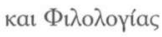

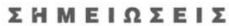

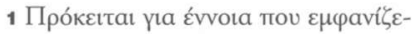

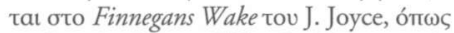

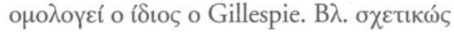

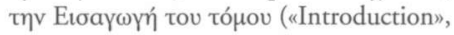

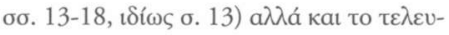

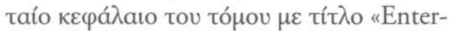

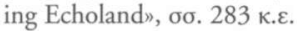

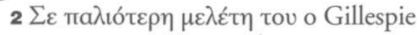




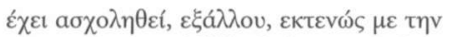

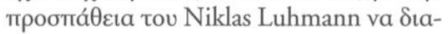

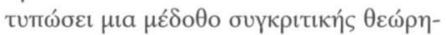

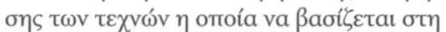

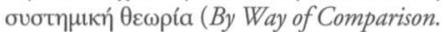
Reflections on the Theory and Practice of Comparative Literature, Paris, Honoré Champion, 2004).

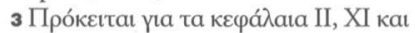
XII.

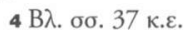

5 В $\lambda$. бо. 175 к.ع.

6 В $\lambda$. бо. 211 к.ع.

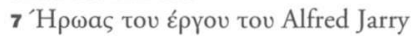
(1873-1907) $\mu \varepsilon$ тí $\lambda_{0}$ Gestes et opinions du docteur Faustroll, pataphysicen (Exploits and Opinions, of Dr. Faustroll, pataphysician),

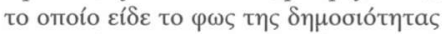

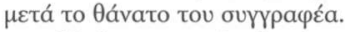

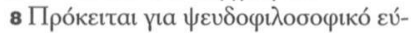

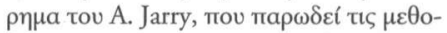

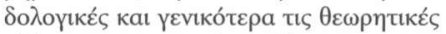

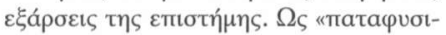

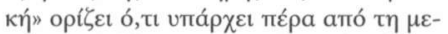

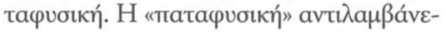

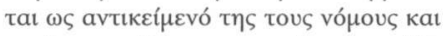

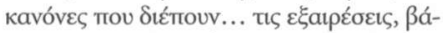

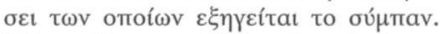

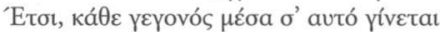

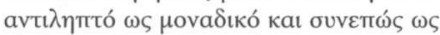

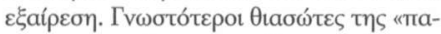

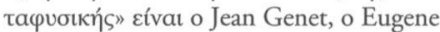
Ionesco, o Raymond Queneau, o Boris Vian к.т.á.

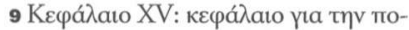

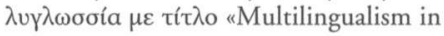

the High Modernist Novel and Poem" ( $\sigma \sigma$.

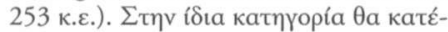

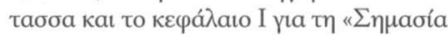

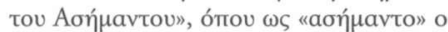

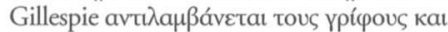

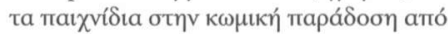

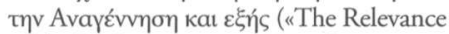
of the Irrelevance", бо. 19 к.ع.).

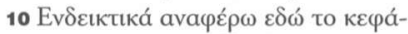
$\lambda$ a

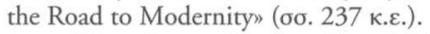

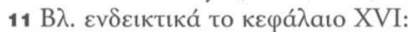
"Portraits of the Artist as a Young Siegfried. Mann's Felix and Joyce's Stephen Approach the Supreme Mysteries» (бо. 261 к.є.).

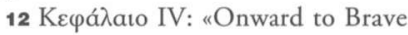

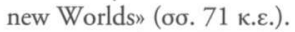

13 Keqádaı VI: "The Fine Art of Erotic Dreaming in Eighteenth-Century

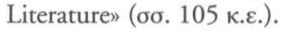

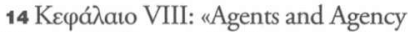
of History in Romantic Literature" (бo. 135 к.ع.).

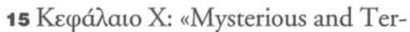
rifying Others since Late Romanticism" (бо. 167 к.ع.).

16 Про́кєıтаı үıа о́ро точ о Gillespie

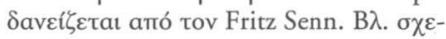

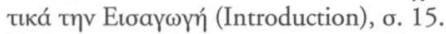

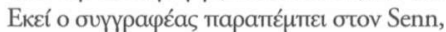

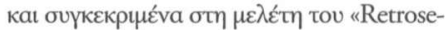
mantics: How Understanding Trails Behind", бто Papers on Joyce, ap. 7/8 (2001-2002), бо. 7-43.

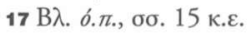

